\title{
Was bewirkt die Volkswirtschaftslehre?
}

\author{
Bruno S. Frey* \\ Universität Zürich
}

\section{Einleitung}

Ökonomen besitzen ein hohes Selbstvertrauen. Sie halten ihre Wissenschaft wegen ihrer analytischen Denkweise und hohen Formalisierung für die Königin der Sozialwissenschaften und nehmen deren Bedeutung für die Lösung gesellschaftlicher Fragen als selbstverständlich an. Gerade weil die Wichtigkeit der Volkswirtschaftslehre beinahe axiomatisch unterstellt wird, werden selten konkrete Beispiele für den Einfluss der Volkswirtschaftslehre auf die Wirtschaft und Gesellschaft angeführt. Vage Hinweise auf Beratungstätigkeit von Ökonomen werden als genügender „Beweis“ angesehen. Wenn es um den Einfluss auf wirtschaftliche und politische Ergebnisse geht, werden Ökonomen entweder sprachlos oder ersetzen Argumente durch blosse Behauptungen.

Das tiefe Niveau der Diskussion um den Einfluss der Volkswirtschaftslehre kontrastiert mit der sonst üblichen Stringenz ökonomischer Argumentation. Wissenschaftlichen Kriterien genügende Analysen des Einflusses der Volkswirtschaftslehre auf die Gesellschaft fehlen weitgehend. ${ }^{1}$ So wird zum Beispiel allzu häufig Input mit Output verwechselt, etwa wenn Beratungstätigkeit mit der Wirkung der Beratung gleichgesetzt wird. Derartig elementare Fehler wären in andern Bereichen der Ökonomie undenkbar. Dass Ökonomen zur Beratung herangezogen werden, ist unbestritten, deren Wirkung bleibt jedoch offen. Ökonomische Beratung kann die gesellschaftliche Wohlfahrt verbessern; möglicherweise ist sie aber unwirksam oder verschlechtert sie sogar.

In diesem Beitrag werden mögliche Ansatzpunkte für eine systematische

* Bruno S. Frey ist Professor an der Universität Zürich, Institut für Empirische Wirtschaftsforschung, Blümlisalpstr. 10, 8006 Zürich, Tel. 01/634 37 31, Fax. 01/634 49 07, e-mail bsfrey@iew.unizh.ch. Für hilfreiche Hinweise zu einer früheren Fassung danke ich Charles Beat Blankart, Gottfried Bombach, Reiner Eichenberger, René L. Frey, Gérard Gäfgen, Reto Jegen, Gebhard Kirchgässner, Stephan Meier, Ewald Nowotny, Margit Osterloh, Friedrich Schneider und Hannelore Weck-Hannemann.

1. Vgl. auch Nelson (1987), S. 49; Cordes, Klamer und Leonard (1993), S. 462; Middleton (1998); Klein (1999). 


\section{Bruno S. Frey}

Analyse des Einflusses der Volkswirtschaftslehre aufgeführt und es wird auf einige empirische Belege hingewiesen. Grundsätzlich wird dafür die ökonomische Denkweise angewandt; es wird von Angebot und Nachfrage, Mengen und Preisen, Inputs und Outputs und Gleichgewichten auf Märkten für ökonomische Ideen und Ökonomen die Rede sein. Wie deutlich werden wird, kann keine Rede von einer geschlossenen Theorie und überzeugenden Empirik sein; das Gebiet der "Ökonomik der Ökonomik" befindet sich erst am Anfang. Der Beitrag geht über eine Analyse der wirtschaftspolitischen Beratung im engeren Sinne hinaus, weil in dieser Literatur der Erfolg der Beratung selten systematisch behandelt wird. Im übrigen steht eine reichhaltige Literatur über den Beratungsprozess und dessen Institutionen zur Verfügung. ${ }^{2}$

In Abschnitt 2. werden die grundsätzlichen Möglichkeiten zur Erfassung des Einflusses aufgeführt. Im folgenden Abschnitt wird diskutiert, ob sich eine Produktionsfunktion zwischen Volkswirtschaftslehre als Input und gesellschaftlichen Ergebnissen als Output formulieren lässt. In Abschnitt 4. wird der Einfluss von Ideen und in Abschnitt 5. von Personen erörtert. Der folgende Abschnitt beschäftigt sich mit Ökonomen als Politikern. Abschnitt 7. enthält eine abschliessende Einschätzung aus persönlicher Sicht.

\section{Wie lässt sich der Einfluss der Volkswirtschaftslehre erfassen?}

Zumeist wird ohne weitere Überlegung einfach behauptet, die Volkswirtschaftslehre sei für die Gesellschaft von grosser Bedeutung. Berühmt ist Keynes' Feststellung, wonach vor allem ökonomische Ideen die Welt regieren ${ }^{3}$ - wobei allerdings dieser überragende Einfluss mit den politischen Philosophen (!) geteilt werden muss. Fünfzig Jahre später fühlt sich Hahn (1985) zu einer „Lobpreisung der ökonomischen Theorie" berufen, und ähnliche enthusiastische Einschätzungen sind häufig zu lesen und zu hören. Etwas zurückhaltender sind jene Ökonomen (z.B. Krugman 1996, Schultze 1996, S. 31), welche die Hauptleistung der Volkswirtschaftslehre darin sehen, die von Politikern geplanten „Riesenfehler“ zu verhindern.

Es gibt jedoch auch ganz gegensätzliche Ansichten. Der Economist (1997, S. 13) beklagt den „Puzzling Failure of Economics“ und der New Yorker (Cassidy 1996, S. 50-1) meint: „.. . a good deal of modern economic theory, even the kind

2. Dabei hat sich die deutschsprachige Volkswirtschaftslehre besonders ausgezeichnet, beispielshaft etwa Jöhr und Singer (1955), von Beckerath und Giersch (1963), Schneider (1968), Borner (1975), Gäfgen (1976), Bonus (1982), Meier und Mettler (1988), Kirchgässner (1996, 1998, 1999), R. L. Frey (1996). Vgl. auch Peacock (1992), Peters und Barker (1993) und Wyplosz (1999).

3. "(T)he ideas of economists and political philosophers ... are more powerful than is commonly understood. Indeed the world is ruled by little else" (Keynes 1936, S. 383-4). Hayek (1991, S. 37) schliesst sich dieser Meinung an, fügt jedoch hinzu: „... economists have this great influence only in the long run and only indirectly". 


\section{Was bewirkt die Volkswirtschaftslehre?}

that wins Nobel Prizes, simply does not matter much“. Die meisten führenden Ökonomen seien ausserhalb ihres Ghettos weitgehend unbekannt und kaum eines ihrer Bücher ziehe Aufmerksamkeit auf sich. Die Politiker kümmerten sich immer weniger um akademische Ökonomen und liessen sich von Vertretern anderer Wissenschaften beraten. Tatsächlich wird diese Ansicht auch von einigen Insidern vertreten, etwa von Galbraith (1975, S. 12), „The economic profession - I choose my words with care - is intellectually bankrupt. It might as well not exist". Aber auch von der ideologisch genau entgegengesetzten Seite tönt es ähnlich kritisch: „We economists in recent years have done vast harm to society at large and to our profession in particular by claiming more than we can deliver" (Friedman 1972, S. 12). Clower (1989, S. 23), ein ehemaliger Herausgeber der American Economic Review, geht noch weiter: „Much of economics is so far removed from anything that remotely resembles the real world that it's often difficult for economists to take their own subject seriously". Scheinbar erstaunt stellt Stiglitz (1998, S. 5) fest, dass sich die Denkweisen in der Umgebung des Weissen Hauses, in dem er als Chairman des Council of Economic Advisors tätig war, diametral von der ökonomischen Logik unterscheiden und fragt beinahe verzweifelt: „Why is it so difficult to implement even Pareto improvements?" Am härtesten urteilt der Dogmenhistoriker und Wissenschaftstheoretiker Blaug (1997, S. 2), wenn er feststellt: „... modern economics is sick; economics has increasingly become an intellectual game played for its own sake and not for its practical consequences."

Neben diesen positiven und negativen Einschätzungen des Einflusses der Ökonomie gibt es noch eine dritte Auffassung, nämlich dass ein Einfluss weder möglich noch zu erwarten sei. Die Wirtschaft befindet sich gemäss der ökonomischen Theorie à la Chicago in einem effizienten Gleichgewicht. Infolgedessen können Ökonomen für die Politik nichts Nützliches beitragen, und wenn sie es dennoch zu tun glauben, unterliegen sie einem Irrtum (Stigler 1982, Becker 1985, Wittman 1989).

Die angeführten Behauptungen sind zwar amüsant zu lesen und vermögen vielleicht wichtige Aspekte aufzuzeigen, sie beruhen aber bestenfalls auf einem mehr oder weniger informierten Gefühl. Auch das Eigeninteresse mag mitspielen; es gibt Verteidiger des Status quo und Erneuerer. Allerdings ist eine Zuordnung nicht einfach. So gibt es mit dem Nobelpreis gekrönte Ökonomen, die dennoch ihre eigene Wissenschaft hart kritisieren, wie etwa Leontief (1971) oder Vickrey (vgl. Cassidy 1996, S. 50). Um Klarheit zu schaffen, ist daher eine analytisch fundierte Betrachtung notwendig, die $\mathrm{zu}$ empirisch testbaren Hypothesen führt.

In diesem Aufsatz werden drei grundsätzliche Ansatzpunkte unterschieden, wie sie in Figur 1 dargestellt sind:

1) Eine Produktionsfunktion, die einen systematischen, aber hochaggregierten Zusammenhang zwischen dem Input der Volkswirtschaftslehre und dem Output in Form des Zustands der Wirtschaft und Gesellschaft herzustellen versucht. 
Bruno S. Frey

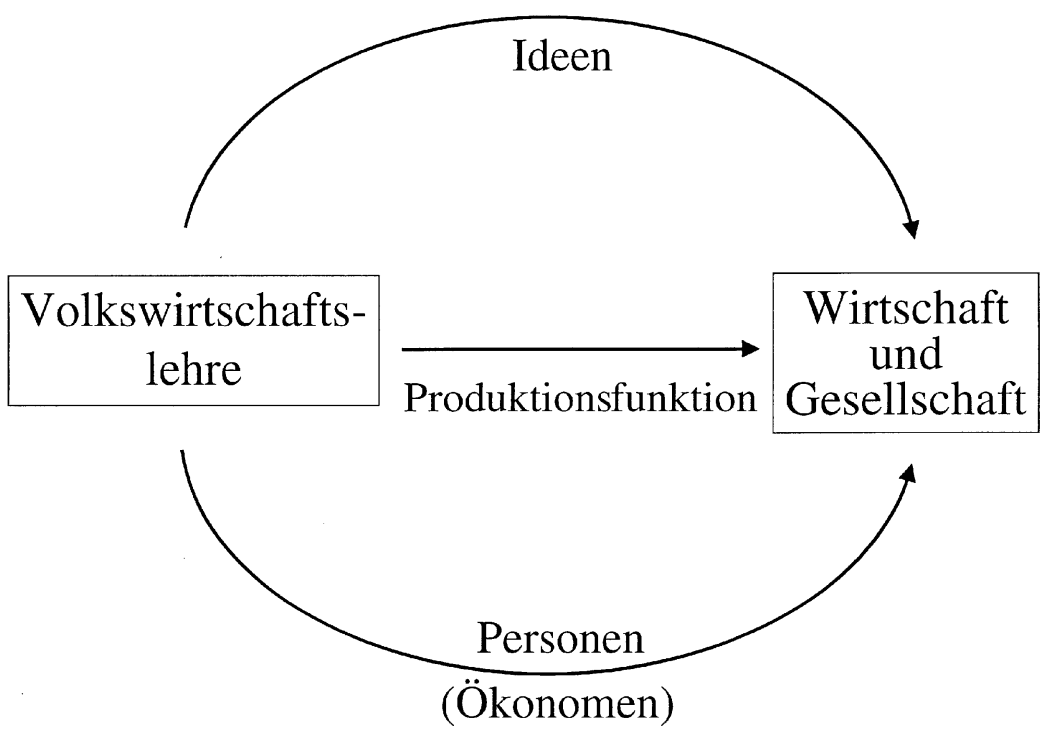

Figure 1 Drei Ansatzpunkte zur Erfassung des Einflusses der Volkswirtschaftslehre

Die zwei andern Ansatzpunkte konzentrieren sich auf spezifische Bündel von Transmissionswegen.

2) Der Einfluss mittels ökonomischen Ideen. Was sind überhaupt „ökonomische Ideen“? Sind sich die Ökonomen darüber einig? Auch ist $\mathrm{zu}$ analysieren, wie die Transmission der Ideen $\mathrm{zu}$ Ergebnissen vor sich geht. Als direkte und indirekte Einflusskanäle ökonomischer Ideen lassen sich unterscheiden: (2.1) Die interne Wissenschaftstätigkeit mittels akademischen Publikationen; (2.2) Der Einfluss auf andere Wissenschaften; (2.3) Der Einfluss über die Medien; (2.4) Die Anwendungen der Volkswirtschaftslehre in der Wirtschaftspolitik, d.h. die Umsetzung „typisch ökonomischer" Vorschläge und (2.5) die vertiefte Untersuchung ausgewählter Episoden und Bereiche der Politik.

3) Einfluss durch Personen (Ökonomen). ${ }^{4}$ Es lassen sich folgende Einflusskanäle unterscheiden: (3.1) Ausbildung von Ökonomen; (3.2) Zahl und Entlohnung der ökonomischen Wissenschaftler; (3.3) Marktnachfrage nach Ökonomen ausserhalb der Universität, wobei wiederum die Zahl und das Einkommen der Beteiligten betrachtet werden kann; (3.4) Beratungsinstitutionen, neben den Universitäten die Think Tanks, private Firmen etc.; (3.5) Ökonomen als Politiker.

4. Allein zur einfacheren Lesbarkeit werden unter dem Begriff „Ökonomen“ immer Personen beiden Geschlechtes verstanden. 


\section{Was bewirkt die Volkswirtschaftslehre?}

Der Einfluss ökonomischer Ideen und von Ökonomen unterscheidet sich: Ökonomische Ideen können auch durch Nichtökonomen vorgebracht werden und Ökonomen können auch (und tun dies auch häufig) nichtökonomische Ideen vertreten.

Insgesamt ergeben sich zwölf Ansatzpunkte (wenn die bereits angeführten eher impressionistischen Behauptungen berücksichtigt werden). Wie deutlich werden wird, sind viele dieser Ansätze noch kaum ernsthaft analysiert worden.

\section{$\underline{\text { 3. Produktionsfunktion }}$}

Die Volkswirtschaftslehre (V) als Input bewirkt als Output eine Veränderung des Zustandes von Wirtschaft und Gesellschaft (X), der natürlich auch durch weitere Einflüsse $(Z)$, sowie durch andere Wissenschaften (A) beeinflusst wird:

$$
\mathrm{X}=\mathrm{F}(\mathrm{V}, \mathrm{Z}, \mathrm{A}) \text {. }
$$

Der Zustand der Wirtschaft und Gesellschaft X lässt sich durch verschiedenste Aspekte charakterisieren, etwa durch das Niveau oder die Wachstumsrate des Prokopfeinkommens, die Arbeitslosenquote, die Inflationsrate, den Aktienwert aller kotierten Firmen, die subjektive Einschätzung des Wohlbefindens oder Glücks (vgl. Frey und Stutzer 1999), das Ausmass an Innovationen etc. Der Input der Volkswirtschaftslehre kann mit der Zahl, dem Anteil oder dem Wachstum akademisch oder in der Praxis, Politik und Verwaltung tätiger Ökonomen oder der Menge und Qualität der Forschung anhand von Publikationen und Zitierungen gemessen werden (vgl. dazu Abschnitte 4. und 5.). Der Input durch andere Wissenschaften (Variable A) lässt sich durch die entsprechenden Zahlen etwa für die Politikwissenschaft, Soziologie oder Rechtswissenschaft erfassen.

Über die Art des funktionalen Zusammenhangs gibt es die unterschiedlichsten Vorstellungen. Gemäss Allen (1979, S. 18-19) gilt: „... a country's economic progress is in inverse proportion to the distinction of its economists“. Wyplosz (1999, S. 60) meint: „The economic successes of France and Japan seem to indicate that economic policy can be carried out perfectly well without economists“. Stigler (1982, S. 63) glaubt: „Economists exert a minor and scarcely detectable influence on the societies in which they live", und hinsichtlich Publikationen behauptet Tullock (1984, S. 229), „... the average article in economic journals these days has very little prospect of contributing to the well-being of the world".

Empirische Schätzungen der Produktionsfunktion der Volkswirtschaftslehre gibt es bisher nur wenige. Barro (1993) hat ironisierend folgenden Zusammenhang zwischen den Ökonomen im Council of Economic Advisors und der Wirtschaftslage geschätzt:

... economic outcomes (measured by the contribution to the misery index) and the credentials of the chairman of the council (measured by citations count) are 


\section{Bruno S. Frey}

essentially uncorrelated. Although some who are highly ranked on citations ... do well on performance, the highly ranked Mr. Schultze ends up with the worst economic outcomes. Moreover, some of the chairmen who ranked low on citations ... emerge with good economic performance.

Magee (1992) hat eine vergleichbare Produktionsfunktion für Juristen anhand einer Querschnittsuntersuchung für 54 Länder ökonometrisch geschätzt. Nach Berücksichtigung weiterer Determinanten $(Z)$ zeigt sich, dass Juristen einen umgekehrt U-förmigen Einfluss auf das Wirtschaftswachstum ausüben, d.h. es gibt eine optimale Juristendichte. $\mathrm{Zu}$ viele Juristen produzieren schwerwiegende negative externe Effekte.

Wesentlich weiter fortgeschritten ist die ökonometrische Schätzung der „spillovers“ der Universitätsforschung auf kommerzielle Innovationen, wie sie etwa von Jaffe (1989) und Acs, Audretsch und Feldman (1991) vorgenommen wurden. Verwandt ist auch die Analyse der Frage, ob das Studium der Volkswirtschaftslehre kooperatives Verhalten beeinträchtigt und damit die Bereitstellung öffentlicher Güter und die Überwindung negativer externer Effekte erschwert. Frank, Gilovich und Regan (1993) schliessen auf Grund von Experimenten, eigennütziges und zynisches Denken und Verhalten zeige sich bei Studenten der Volkswirtschaftslehre häufiger als bei andern Studienrichtungen und verstärke sich im Ablauf des Ökonomiestudiums. Einige dieser Unterschiede können jedoch auch auf Selbstselektion zurückzuführen sein, d.h. zum vorneherein eher egoistische und zynische junge Leute entschliessen sich vorzugsweise für das Studium der Ökonomie. ${ }^{5}$

Die Verwendung einer Produktionsfunktion zur Erfassung des Einflusses der Volkswirtschaftslehre hat drei wichtige Vorzüge: (1) Die Aufmerksamkeit wird auf den Unterschied zwischen Output und Input der Volkswirtschaftslehre gelenkt, eine wichtige Unterscheidung, die ansonsten allzu häufig vernachlässigt wird; (2) Eine Produktionsfunktion zwingt zu einer systematischen Betrachtung der Beziehung zwischen Input und Output der Volkswirtschaftslehre, ist elegant und entspricht der ökonomischen Denktradition; (3) Alle Einflusskanäle werden prinzipiell zugelassen, so werden z.B. sowohl mikroals auch makroökonomische Determinanten erfasst.

Eine Produktionsfunktion zwischen ökonomischen Inputs und Outputs ist jedoch mit schwerwiegenden Problemen konfrontiert: (1) Die Voraussetzungen für die Schätzung einer Produktionsfunktion sind vermutlich nicht erfüllt: „the market [for economics] at the production possibility frontier is inefficient in the sense that, given the output of pure theory, we could achieve a better output of applied work. We are not at the production possibility frontier" (Mayer 1993, S. 10); (2) Die genaue Spezifikation der Produktionsfunktion ist willkürlich, so sind etwa die Zeitverzögerungen, innerhalb denen eine Wirkung der Volkswirtschaftslehre zu erwarten ist, nicht festgelegt; ${ }^{6}$ (3) Besonders

5. Carter und Irons (1991); Frey, Pommerehne und Gygi (1993); Laband und Beil (1999).

6. Schelling (1997, S. 146) etwa spricht von einer Zeitverzögerung von zwei Jahrzehnten, bis ökonomische Vorschläge in die Umweltpolitik Eingang fanden. 


\section{Was bewirkt die Volkswirtschaftslehre?}

schwerwiegend ist die ungelöste Kausalität. Es ist durchaus möglich, dass nicht die Volkswirtschaftslehre die Wirtschaft und Gesellschaft beeinflusst, sondern dass umgekehrt die Volkswirtschaftslehre herbeigerufen wird, wenn wirtschaftliche und gesellschaftliche Probleme entstanden sind. Aus diesem Grund ist nicht einmal das erwartete Vorzeichen der Korrelation eindeutig; (4) Eine Variable könnte gemeinsam die Volkswirtschaftslehre und die Gesellschaft beeinflussen.

Insgesamt handelt es sich bei der Produktionsfunktion der Volkswirtschaftslehre um einen typischen Fall einer Messung ohne ausreichender Theorie. Aus diesem Grund führt kein Weg daran vorbei, den Einflusses der Volkswirtschaftslehre vertiefter zu analysieren.

\section{Einfluss ökonomischer Ideen}

\subsection{Ein ökonomischer Konsens?}

Gibt es überhaupt typisch „ökonomische Ideen“, die sich systematisch von denjenigen anderer Wissenschaften und Laien unterscheiden und die von der Mehrzahl der Ökonomen akzeptiert werden? Der bekannte amerikanische akademische Politikberater Schultze (1996, S. 26) ist sich dessen sicher: „There is a distinct consensus among economists". In letzter Zeit ist der „Washingtoner Konsens" (Williamson 1994) in aller Munde. Er wird gar als „universelle Konvergenz" gesehen und umfasst nichts weniger als den "common core of wisdom embraced by all serious economists ..." (vgl. Middleton 1998, S. 344). Er besteht vor allem in einer aktiven Wirtschaftspolitik zur Beseitigung mikroökonomischer Verzerrungen und der Herstellung eines makroökonomischen Gleichgewichtes (vor allem mittels Budgetausgleich). Die Vorstellung „economics (is) after all a science capable of truth and progress" (Williamson 1997, S. 365) widerspricht allerdings der Auffassung, wonach sich Zyklen von individualistischen und kollektivistischen Ideen und Politiklösungen immer wieder ablösen (Hirschman 1982). Sie widerspricht auch Brittans (1995, S. 21) Feststellung „the idea of technocratic value free economics has had its day".

Das Ausmass an tatsächlich existierendem Konsens unter professionellen Ökonomen ist Gegenstand mehrerer empirischer Untersuchungen. ${ }^{7}$ Sie stellen zwar einen Konsensbereich fest, gleichzeitig finden sie jedoch erhebliche Unterschiede in den Auffassungen, die teilweise historisch und institutionell

7. Für das Vereinigte Königreich Brittan (1973); für die USA Kearl, Pope, Whiting and Wimmer (1979), sowie Alston, Kearl and Vaughan (1992); für Deutschland Schneider, Pommerehne und Frey (1983); für Oesterreich Pommerehne, Schneider und Frey (1984); für die Schweiz Frey, Pommerehne, Schneider und Weck (1982); für Europa Frey, Pommerehne, Schneider und Gilbert (1984). Van Dalen und Klamer (1997) diskutieren unterschiedliche Schulen in den Niederlanden und Fuchs, Krueger und Poterba (1997) und Samuels (1980) erforschen den Konsens hinsichtlich finanz- und aussenwirtschaftlichen Gebieten. 


\section{Bruno S. Frey}

begründet sind: Beispielsweise beurteilten französische und österreichische Ökonomen in den 80er Jahren Staatsinterventionen weit positiver als amerikanische, deutsche und schweizerische Ökonomen.

\subsection{Verbreitungsprozess}

Es gibt verschiedene Vorstellungen über den Weg von den ökonomischen Ideen zur (möglichen) Wirkung auf die Wirtschaft und Gesellschaft (vgl. dazu auch Coats und Colander 1989).

(a) Problemlose Übertragung. Dieser Auffassung zufolge lässt sich die Kluft zwischen ökonomischen Ideen und deren Anwendung überwinden, wenn die wissenschaftlichen Erkenntnisse in einer für Nicht-Ökonomen verständlichen Sprache vorgebracht werden (z.B. Hamilton 1992, Blendon et al. 1997). Allerdings ist der Markt für diese Transformation nur wenig ausgeprägt (Cordes, Klamer und Leonard 1993, S. 467). Die Distanz lässt sich nicht so einfach überbrücken, wie die bekannte ökonomische Beraterin Rivlin (1987, S. 1) feststellt: „Economists and political leaders not only miscommunicate, but each accuses the other of incompetence, obfuscation, self-serving motives, and anti-social behavior". McCloskey (1983) und Klamer und Colander (1990) argumentieren, dass es um völlig unterschiedliche Diskurse geht, die zum Teil sogar unvereinbar sind.

(b) Automatischer Prozess. Die Übertragung ökonomischer Ideen auf die Gesellschaft verläuft wie eine ansteckende Epidemie, deren Verlauf nur wenig beeinflussbar ist und einer exogen vorgegebenen maximalen Verbreitung zustrebt. Diese mechanistische Vorstellung ist unhaltbar, insbesondere weil völlig offen bleibt, welche Ideen diesen Siegeszug antreten.

(c) Informationstheorie. Die ökonomischen Ideen werden von einer Quelle den professionellen Ökonomen - ausgeschickt und verschlüsselt. Im Transmissionsprozess wird diese Information durch irrelevante oder falsche Nachrichten gestört. Die Empfänger - die Laien, Politiker und Bürokraten entschlüsseln schliesslich die Information. Dieser Sicht folgend ist der Übertragungsprozess durch manche Irrtümer und Missverständnisse geprägt.

(d) Markt für ökonomische Ideen. Die Anbieter - neben den akademischen Ökonomen auch Forschungsinstitute, Think Tanks, Stiftungen und profitorientierte Beratungsfirmen (vgl. beispielsweise König 1999) - bieten ökonomisches Wissen an, wenn sie dafür ausreichende Anreize in Form materieller oder immaterieller Entlohnung erhalten. An Universitäten, die dem internationalen Wettbewerb ausgesetzt sind, bestehen allerdings nur geringe Anreize, sich dabei mit gesellschaftlichen Gegenwartsproblemen zu beschäftigen. Karrierefördernd ist vielmehr eine formal möglichst weit getriebene Auseinandersetzung mit den innerhalb des Faches selbst definierten Problemen. Wie es Colander (1989, S. 34) lapidar formuliert: „The incentives in the economics profession are for articles, not ideas“. Die (potentiellen) Nachfrager wägen den Nutzen der Information mit den Kosten für deren Erwerb, Verarbeitung und praktischen Nutzung ab. Eine 


\section{Was bewirkt die Volkswirtschaftslehre?}

verständlichere Darlegung vermindert diese Kosten nur marginal; daneben beeinflussen eine grosse Zahl anderer Faktoren die Intensität der Nachfrage nach ökonomischem Wissen. Eine ökonomische Betrachtung erlaubt, die verschiedenen Aspekte des Übertragungsprozesses systematisch zu ordnen, die Kausalitäten mittels einer Verhaltensanalyse $\mathrm{zu}$ bestimmen, die sich einstellenden Gleichgewichte $\mathrm{zu}$ charakterisieren und empirisch testbare Hypothesen abzuleiten. Dieses Forschungsgebiet ist jedoch noch kaum beackert. ${ }^{8}$ Dazu gehört auch die Analyse des Einflusses der Medien für den Transformationsprozess. Diese nehmen offensichtlich einige ökonomische Ideen auf (vor allem wenn sie kontrovers sind, vgl. Rivlin 1987), vernachlässigen hingegen systematisch andere Informationen von Seiten der Volkswirtschaftslehre. Mit der Berücksichtigung in den Medien ist überdies noch nichts über deren Einfluss auf die Wirtschaft und Gesellschaft ausgesagt. Auch hier stellt sich wieder das Problem der umgekehrten Kausalität.

\subsection{Die Quelle ökonomischer Ideen}

Ökonomen wie auch Nichtökonomen als Einzelpersonen, aber auch Forschungsinstitute wie Think Tanks, Stiftungen und marktliche Beratungsgesellschaften können ökonomische Ideen vorbringen. Der Ursprung besteht in aller Regel in einer wissenschaftlichen Aktivität, die sich in Publikationen äussert und deren Wirkung innerhalb der Wissenschaft mit der Zahl der Zitierungen erfasst wird. Eine weitere Quelle besteht in der akademischen Lehre, von wo aus das Wissen durch die Studierenden in die Praxis vermittelt wird (vgl. den folgenden Abschnitt 4.4.). Schliesslich spielt auch die Popularisierung ökonomischer Ideen in verschiedensten Formen (Zeitungsartikel, Vorträge, Fernsehauftritte) eine Rolle.

Ökonomische Ideen können auch auf Umwegen über andere Wissenschaften einen Einfluss auf Wirtschaft und Gesellschaft ausüben. Das Ausmass des „ökonomischen Imperialismus" (Stigler 1984) ist ein „... important indicator of success of economics" (Demsetz 1997, S. 1). Der Einfluss auf die Politikwissenschaft ist in der Tat stark (zwischen 1977/80 und 1988/90 hat sich die Zahl der Zitierungen ökonomischer Werke verdreifacht), hingegen auf die Soziologie eher bescheiden (zwischen 1970 und 1980 hat die Zitierungszahl zwar deutlich zugenommen, ist aber in den 90er Jahren nicht mehr gestiegen, vgl. Baron und Hannan 1994).

\subsection{Art der Übertragung ökonomischer Ideen}

Gemäss Nelson (1987, S. 65) gilt: „Given almost any social problem, the first and natural response of most professional economists is to look for potential

8. Versuche in dieser Richtung finden sich in Middelton (1998) für das Vereinigte Königreich und allgemein in Frey und Eichenberger (1992, 1993, 1997). 
means of manipulating the market mechanism - to solve the problem by arranging what might be called a planned market". Allerdings hat sich die Auffassung, was der "richtige“ Ratschlag ist, im Laufe der Zeit drastisch geändert. „Half a century ago, economists were quick to favor government ownership of firms as soon as any market inequities or imperfections, such as monopoly power, were even suspected" (Shleifer 1998, S. 133-4). Dazu gehörten gerade auch die prominentesten Ökonomen dieser Zeit, von denen einige wie Arthur Lewis, James Meade und Maurice Allais später mit dem Nobelpreis ausgezeichnet wurden. Die damaligen Vertreter unserer Zunft übersahen den "grotesque failure" der staatlichen Firmen und erkannten nicht, was heute als „richtig“ gilt, nämlich „... that private ownership is the crucial source of incentives to innovate and become efficient" (Shleifer 1998, S. 135).

$\mathrm{Zu}$ den heute als „typisch ökonomisch“ geltenden Vorschlägen zählen hinsichtlich Institutionen die Unabhängigkeit der Notenbank, der Freihandel, die Privatisierung und Deregulierung (die zwei letzteren zählt Nelson $1987 \mathrm{zu}$ den „success stories“) und hinsichtlich Instrumenten marktliche Umweltsteuerung, Gutscheine (vouchers), negative Einkommenssteuer, Road Pricing und Peak Load Pricing (Faulhaber und Baumol 1988), Leistungslöhne und Aktienoptionen (z.B. Prendergast 1999), sowie Auktionen, etwa für Radio- und Fernsehfrequenzen und für die Umweltnutzung (z.B. Joskow, Schmalensee und Bailey 1998), sowie Finanzmarktinstrumente (z.B. Zimmermann 1999). Allerdings sollten dabei Fehlallokationen von ökonomischen Analysen nicht übersehen werden. So beklagt etwa Blinder (1997, S. 17) als ehemaliger Vizepräsident der Federal Reserve: „... theorists have lavished vastly too much attention on a nonexistent time-inconsistency problem while ignoring a much more real problem that arises when central bankers ,follow the market' too closely".

Nur wenige Studien untersuchen den Einfluss ökonomischer Ideen anhand von konkreten wirtschaftspolitischen Episoden. Galbraith (1988, S. 224) berichtet: „Council of Economic Advisors (CEA) Chairman Murray Weidenbaum, when asked directly what weight of influence, on a scale of one to ten, economists had enjoyed in drafting the original tax program of the (Reagan) administration, replied, Zero". Aaron (1987, S. 7) spricht von einer „frustrierenden Situation“ bei der amerikanischen Steuerreform von 1986 (unter Präsident Bush), weil sich auch führende Ökonomen über wesentliche Aspekte der erwarteten Wirkung auf Effizienz und Verteilung völlig uneinig waren. Cordes, Klamer und Leonard (1993) stellen ebenfalls fest, dass der Einfluss der ökonomischen Ideen nur gering war. Sie äussern sogar die Vermutung, dass Ökonomen zwar einflussreich waren, nicht aber die Volkswirtschaftslehre (S. 477). Für die Diskussion über die Errichtung der NAFTA kommen Klamer und Meehan $(1999$, S. 1) zum Schluss: „... political arguments crowded out serious economic arguments ... academic economists had no role to play when it came to the final act". Eine ähnliche Aussage hinsichtlich der Reform des amerikanischen Gesundheits- und Wohlfahrtssystem trifft Cassidy 


\section{Was bewirkt die Volkswirtschaftslehre?}

(1996, vgl. auch Krugman 1995). Für Deutschland meint Die Wirtschaftswoche (1997, S. 36), „Der Einfluss der Ökonomen auf die deutsche Wirtschafts- und Finanzpolitik wird immer geringer."

Viele Beobachter sind sich einig, dass nur einfache ökonomische Ideen einen Einfluss auszuüben vermögen. So spricht etwa Blinder (1997, S. 18) von „trained common sense“ (ähnlich z.B. Coase 1999), Krugman (1995, S. 32) von „very simple economic ideas" und Stein (vgl. Hamilton 1992, S. 62), ein früherer Vorsitzender des CEA, meint „most of the economics that is usable for advising on public policy is about the level of the introductory undergraduate course“. Bemerkenswerterweise hat schon Marshall (1890, S. 779) die gleichen Erfahrungen gemacht.

\section{$\overline{\text { 5. Einfluss von Ökonomen }}$}

Der Einfluss von Ökonomen als Personen kann als ein Markt verstanden werden. ${ }^{9}$ Zuerst werden das Angebot und die Nachfrage erörtert. Dann werden die Preise in Form der erzielten Einkommen als Gleichgewichtsphänomene analysiert. Abschliessend wird erörtert, von welchen Bedingungen der Einfluss der Ökonomen abhängt.

\subsection{Angebot}

Ökonomen können als Praktiker direkt die Gesellschaft beeinflussen. Sie können in der staatlichen Verwaltung (vgl. Coats 1981, 1989), in internationalen Organisationen (Coats 1986), in öffentlichen oder privaten Forschungsinstituten oder in Unternehmungen tätig sein und ihr Wissen einbringen. ${ }^{10}$ Sie können auch als Berater oder als Einzelperson in öffentlichen Institutionen wie dem deutschen Sachverständigenrat (Schlecht und van Suntum 1995), den schweizerischen „Drei Weisen“ (Weber 1982) oder dem amerikanischen Council of Economic Advisors (Stein 1996, Schultze 1996, Feldstein 1997, Stiglitz 1997), in wissenschaftlichen Beiräten zu Ministerien, in Kommissionen und in privaten Beratungsfirmen aktiv sein. In den USA haben letztere in den vergangenen Jahren ein „explosives Wachstum“ (Mandel 1999, S. 115) zu verzeichnen. Hinsichtlich der Qualität der Beratung vermutet Wyplosz (1999, S. 54), „... the field of policy advice draws some of the best, but also some of the worst, academic economists". Einen indirekten Einfluss können akademische Ökonomen auch mittels ihrer Lehr- und Forschungstätigkeit ausüben.

Die verschiedenen angeführten Tätigkeiten können sich fundamental voneinander unterscheiden. Im einen Extrem sollten die Ökonomen

\footnotetext{
9. Wie Hansen (1999, S. 147) feststellt, wird auch der Arbeitsmarkt für Ökonomen von Ökonomen kaum systematisch analysiert.

10. Ihre Tätigkeit als Politiker wird in Abschnitt 6. behandelt.
} 


\section{Bruno S. Frey}

idealerweise als „unparteiische Vertreter des Allgemeinwohls“ tätig sein, im andern Extrem sollten sie im Gegenteil einer bestimmten Auffassung zum Sieg verhelfen. In der amerikanischen Terminologie der forensischen Ökonomie (vgl. das Journal of Forensic Economics) werden sie als „hired guns“ bezeichnet. Das rasche Wachstum dieses Beratungszweiges ${ }^{11}$ geht auf die rapide Zunahme von Übernahmen und Zusammenschlüssen von Firmen, die Deregulierung und Privatisierung bisher staatlicher Tätigkeiten und die zum Teil enormen Forderungen bei Gerichtsfällen (z.B. Schadensersatzklagen) zurück. Die Gefahr einer zugunsten von Parteiinteressen verzerrten Analyse ist offensichtlich, besonders wenn den beteiligten Ökonomen ein erfolgsorientierter Leistungslohn bezahlt wird (Thornton und Ward 1999). Allerdings gibt es deutliche Schranken (Posner 1999), Ehrlichkeit und professionelle Kompetenz werden auf diesem Markt geschätzt, weshalb es sich lohnt, eine entsprechende Reputation aufzubauen. Überdies werden die vorgebrachten Argumente von der Gegenseite intensiv überprüft - wegen der direkten monetären Konsequenzen wohl noch systematischer als im Gutachterprozess einer wissenschaftlichen Zeitschrift. Die negativen Aspekte liegen eher in der Vertraulichkeit der erarbeiteten empirischen Daten, was den Fortschritt der Wissenschaft hemmt, sowie in der Festlegung von Wissenschaftlern auf einmal eingenommene Positionen („lock-in effect“).

Ein an der Hochschule tätiger Ökonom ist mit einem deutlich spürbaren trade-off nicht nur hinsichtlich der Zeit, sondern auch der Spezialisierung konfrontiert. Nur außerordentlich begabte Personen können die unterschiedlichen Tätigkeiten erfolgreich miteinander verbinden. Der geniale Keynes ist sicherlich das hervorragendste Beispiel. Heute könnten Larry Summers (gegenwärtig amerikanischer Finanzminister), Joseph Stiglitz (gegenwärtig Chefökonom der Weltbank) oder Otmar Issing (gegenwärtig Mitglied des Direktoriums der Europäischen Zentralbank) genannt werden, die auch weiterhin wissenschaftlich tätig sind.

Ein seinen Eigennutzen verfolgender Ökonom wird die Grenznutzen und Grenzkosten verschiedener Tätigkeiten abwägen. Seine Anreize für eine direkte Beeinflussung von Wirtschaft und Gesellschaft bestehen in extrinsischen Motivatoren wie materieller Entlohnung oder immaterieller Belohnung mittels Ansehen und Macht, aber auch in der intrinsischen Befriedigung durch eine derartigen Tätigkeit selbst. Parteilich orientierte (forensische) Beratungstätigkeiten von guten Ökonomen werden sehr gut honoriert - hier greift Franks (1995) Superstar-Argument. Die Anreize, in wissenschaftlichen Gremien tätig zu werden, sind schwächer und bestehen vor allem im dadurch erworbenen Ansehen und einer gewissen Prominenz (dies dürfte für den Sachverständigenrat und den Beiräten zu den verschiedenen Ministerien wichtig sein). Die Anreize zu einer entsprechenden Tätigkeit sind heute für Personen, die

11. Nach Mandel (1999:115) gilt für den Umsatz: „, . by the scale of academic economics it is an enormous amount ... it is almost certainly larger than the payroll for the full-time faculty of the top 25 economics departments." 


\section{Was bewirkt die Volkswirtschaftslehre?}

ernsthaft eine akademische Karriere verfolgen, nur gering oder gar negativ (Frey und Eichenberger 1993; vgl. auch die von R. L. Frey und B. S. Frey 1995 herausgegebene Sondernummer des Kyklos). Die zeitlichen Möglichkeiten und zu erwerbenden Fähigkeiten müssen vollumfänglich dem Versuch gewidmet werden, in guten wissenschaftlichen Zeitschriften $\mathrm{zu}$ veröffentlichen und möglichst häufig zitiert $\mathrm{zu}$ werden. ${ }^{12}$

\subsection{Nachfrage}

Die Nachfrage nach ökonomischem Wissen äussert sich an verschiedenen Orten, am deutlichsten aber bei der Ausbildung auf universitärem Niveau. In den USA ist die Zahl der Volkswirtschaft als Hauptfach Studierenden zwischen 1992 und 1996 um 30 Prozent zurückgegangen (Siegfried 1998). In der gleichen Periode ist die Zahl der das Doktoratsstudium neu Aufnehmenden um 18 Prozent gesunken. Ein beträchtlicher Anteil der Absolventen amerikanischer Ph.D.-Programme wird exportiert (Siegfried und Stock 1999, S. 116-118). Viele, wenn nicht die meisten, Ph.D.-Programme an amerikanischen Universitäten wären ohne den hohen Ausländeranteil nicht mehr durchzuführen. Ähnliches berichtet Middleton (1998, S. 360) für das Vereinigte Königreich. Volkswirtschaftliche Abteilungen im deutschen Sprachraum sind wegen eines Mangels an Studierenden von der Auflösung bedroht, vor allem weil mit den Studentenbergen in der Betriebswirtschaft verglichen wird. Aus dieser Sicht betrachtet, wird das heutige Studium der Volkswirtschaftslehre von den potentiellen Nachfragern nicht als besonders nützlich angesehen - zumindest wenn mit dem zu betreibenden Aufwand verglichen wird.

Auch eine akademische Lehr- und Forschungstätigkeit in Volkswirtschaftslehre scheint an Attraktivität eingebüsst zu haben. Unter Kollegen ist immer häufiger zu hören, dass sich gerade die besten Absolventen für andere Berufe entscheiden; besonders häufig wählen junge Ökonomen mit Spezialisierung in Finanzmarkt-, Arbeitsmarkt- und Ressourcenökonomie eine Beschäftigung ausserhalb der Universität. Der Anteil der im privaten Sektor tätigen Volkswirte hat in den USA im vergangenen Jahrzehnt um nicht weniger als 70 Prozent zugenommen (Siegfried und Stock 1999, S. 129-133). Gerade dadurch wird deutlich, dass die Nachfrager die Ausbildung als für praktische Tätigkeiten nicht ausreichend erachten (vgl. auch Krueger 1999 und die von der American Economic Association und der Royal Economic Society beauftragten Berichte von Krueger et al. 1991, Towse und Blaug 1988). Es wird sogar behauptet, ein Ökonom müsse nach seiner Ausbildung drei bis vier Jahre "gereinigt“ (Cassidy 1996) oder „umprogrammiert“ (Nelson 1987) werden. Dies entspricht auch der Einschätzung der Studierenden selbst. Nur 3 Prozent (!) der graduierten amerikanischen Ökonomen betrachtet es für ihren Studienerfolg als „sehr wichtig“, eine gründliche Kenntnis der Wirtschaft zu haben; hingegen

12. Entsprechende Untersuchungen für den deutschen Sprachraum sind Bommer und Ursprung (1998), für die Schweiz Meier (1994) und für Europa Eichenberger, Arpagaus und Meier (1999). 


\section{Bruno S. Frey}

aber 57 Prozent „exzellente Mathematikkenntnisse“ zu besitzen und gar 65 Prozent „gut formale Probleme lösen zu können“ (Colander und Klamer 1987, S. 100; neuerdings auch van Dalen und Klamer 1997, S. 65 für die Niederlande). Deshalb kann es nicht überraschen, dass für die Vereinigten Staaten empirisch eine zunehmende Unzufriedenheit der Studierenden mit der Volkswirtschaftslehre festgestellt wird (Siegfried und Stock 1999, S. 130). Dieses Ergebnis dürfte auch für manche andere Länder zutreffen, insbesondere für diejenigen, die sich am stärksten dem amerikanischen Vorbild in der Ökonomieausbildung angenähert haben. In den USA wurden daraus kaum irgendwelche Folgerungen für die Ausbildung in Volkswirtschaftslehre gezogen. Eine Reaktion erfolgt aber von ausserhalb, indem „business and public policy schools ... begin training their own Ph.D.'s rather than drawing heavily on the output of economics departments" (Krueger 1999, S. 155).

Für ein umfassendes Bild müssen die Bestimmungsgründe der Nachfrage vertieft betrachtet werden. Folgende drei Aspekte sind besonders wichtig:

1) Die Nachfrage nach Ökonomen wird durch die Volkswirtschaftslehre zum Teil selbst bestimmt. Wirtschaftstheorien, die staatliche Eingriffe befürworten, erhöhen die Nachfrage nach Ökonomen. Nach Friedman (1986, S. 8-9) wurde die keynesianische Theorie so rasch aufgenommen, weil „... it opened up such wonderful opportunities for employment and influence by economists ... the New Deal was the greatest employment program for economists that ever existed".

2) Das Gleichgewicht auf dem Ökonomenmarkt wird entscheidend durch politische Einflüsse mitbestimmt, insbesondere weil ein erheblicher Teil von ihnen im öffentlichen Sektor tätig sind. Deshalb drängt sich eine Politische Ökonomie der Beratung auf. ${ }^{13}$ Ein wichtiges Ziel von Politikern ist an der Macht zu bleiben. In einer autoritären oder diktatorischen Gesellschaft fragen die Politiker ökonomischen Rat nach, der ihre Macht erhält, auch wenn diese den Bürgern nichts nützt oder sogar schadet. In einer gut funktionierenden Demokratie sichern sich die Politiker ihre Position am ehesten, wenn sie auf die Wählerwünsche eingehen.

3) Die Nachfrage nach ökonomischen Ratschlägen ist umso grösser, je weniger die Politiker die Wünsche der Wähler kennen. Deshalb ist diese Nachfrage in repräsentativen Demokratien höher als in direkten. Die Auflösung der "Drei Weisen“ in der Schweiz im Vergleich zum Fortbestehen des Sachverständigenrates in Deutschland und des Council of Economic Advisors in den USA spricht für diese These. Auf jeden Fall finden die Regierenden eine Unterstützung ihrer Politik durch Ökonomen nützlich und verabscheuen Kritik, Konflikte und Provokationen.

13. Vgl. etwa Blankart (1981), Peacock (1988, 1991, 1994), Jones und Cullis (1993), R. L. Frey (1996), Kirchgässner (1993, 1996, 1999), Frey und Kirchgässner (1994). 


\section{Was bewirkt die Volkswirtschaftslehre?}

\subsection{Einkommen}

Marktpreise und Einkommen reflektieren die individuelle Zahlungsbereitschaft und damit die Bewertung einer Tätigkeit durch die Gesellschaft. Allerdings gilt dies nur für einen gut funktionierenden Markt ohne signifikante externe Effekte und monopolistische Strukturen. Für die marktmässige Nachfrage nach Ökonomen in der Praxis oder als parteiliche Berater dürften die Bedingungen für einen derartigen Markt recht gut erfüllt sein: ${ }^{14}{ }, \ldots$ the ability to command large sums for economic expertise helps provide a market-based validation of the worth of studying economics" (Mandel 1999, S. 116). Diese Aussage unterstellt als selbstverständlich, dass Ökonomen keine intrinsische Motivation zur Ausübung ihres Berufes haben.

In andern Bereichen - wie etwa der universitären Tätigkeit - lässt sich kaum von einem gut funktionierenden Markt sprechen, denn der Zugang wird durch verschiedene Schranken begrenzt, es werden (hoffentlich) positive externe Effekte erzeugt und Preise und Einkommen werden administrativ festgelegt. Die erzielten Einkommen reflektieren somit nicht, oder zumindest nicht ausreichend, die Bewertung durch die Gesellschaft.

Die Einkommen von Volkswirten an Universitäten können hingegen dazu verwendet werden, die relative Attraktivität unterschiedlicher Ausbildungszweige zu untersuchen. Dies gilt natürlich nur für Universitäten, welche die Gehälter an die Marktlage anpassen können. Deshalb ist wiederum ein Blick auf die USA nützlich. Die realen Durchschnittseinkommen für Doktoren der Volkswirtschaftslehre sind im Zeitraum 1987-95 um 4,6 Prozent gefallen. Gleichzeitig hat sich die Ausbildungsdauer um 6 Prozent erhöht. Die Rendite der Ausbildung ist somit nach 1987 (wie auch schon zuvor) kontinuierlich zurückgegangen (Siegfried und Stock 1999, S. 132). Das Einkommensdefizit von Volkswirtschaftsdoktoren erstklassiger Universitäten ist im Vergleich zu entsprechenden Juristen und Betriebswirten (in MBA-Programmen) gross und nimmt vermutlich noch zu. Das Einkommen von Volkswirten ist auch im Vergleich zu andern qualifizierten Tätigkeiten, darunter auch Sportlern, Unterhaltungskünstlern und Managern grosser Firmen zurückgegangen (Ehrenberg 1999, S. 137-140).

\subsection{Stärke des Einflusses}

Unter welchen Bedingungen haben Ökonomen den stärksten Einfluss? Aufgrund der gemachten Überlegungen lassen sich einige Bedingungen angeben, die empirisch getestet werden können:

14. Allerdings haben wir schon festgestellt, dass bei der forensischen Tätigkeit einige negative externe Effekte auftreten. Zum Teil handelt es sich um ein reines Verteilungsspiel zwischen den verschiedenen Parteien, das wegen des hohen Aufwandes an menschlichen und sachlichen Ressourcen vermutlich eine deutlich negative Summe erzeugt. 
a) Ein Zeitgeist, sowie Trends, Theorien und Ideologien, die gegen den Markt gerichtet sind und deshalb staatliche Eingriffe befürworten, erlauben Ökonomen einen grösseren Spielraum und mehr Einfluss. Dazu gehören etwa die Planungseuphorie nach dem 2. Weltkrieg und der Keynesianismus. Das Gleiche gilt jedoch auch für Zeiten der Deregulierung und Privatisierung, in denen Ökonomen - aber vor allem auch Juristen - benötigt werden. Ist allerdings eine solche Phase einmal abgeschlossen, geht die Nachfrage nach Volkswirten wieder zurück.

b) Ein legalistisch-interventionistischer Staatstyp ist für Ökonomen günstig. Dazu gehören etwa Frankreich und viele lateinamerikanische Länder.

c) Eine schlechte Wirtschaftslage stärkt die Rolle der Ökonomen, weil dann mehr Staatsinterventionen gefordert und durchgeführt werden.

d) In Situationen fundamentaler Unsicherheit (Siegenthaler 1993) insbesondere beim Zusammenbruch von Wirtschaftssystemen wie in Deutschland nach 1945 und in den Ostblockländern nach 1989 erwarten die Individuen eine Orientierung von Seiten der Wissenschaft. Damit eröffnen sich Handlungs- und Beschäftigungsmöglichkeiten für Ökonomen. Allerdings entsteht dann auch eine erhöhte Nachfrage nach esoterischen Lösungen, zu denen Ökonomen nichts beizutragen haben.

\section{6. Ökonomen als Politiker}

Ein möglicherweise besonders wichtiger Einflusskanal der Volkswirtschaftslehre auf die Wirtschaft und Gesellschaft wurde bisher erst gestreift: Der Einfluss, den Ökonomen unmittelbar im politischen Prozess einnehmen, indem sie in der Exekutive, Legislative oder der Zentralbank Positionen einnehmen (d.h. nicht „nur“ als Berater tätig sind). Allerdings haben einzelne Politiker bei intensivem politischen Wettbewerb wenig Raum für eigene Ideen. Unabhängig von der Ausbildung wird dann jeder Politiker die stimmenmaximierende Politik verfolgen, denn ansonsten verliert er seine Position. Der politische Wettbewerb ist jedoch in aller Regel nicht so stark, als dass nicht spezifisch ökonomische Ideen in den Prozess eingebracht werden könnten.

Welche Ökonomen waren oder sind als Politiker tätig? Für die nachfolgende Aufstellung sind zwei wichtige caveats anzufügen: (1) Über die Wirkung von Ökonomen als Politiker wird nichts ausgesagt. Es lässt sich bestenfalls vermuten, dass je höher die politische Position, desto grösser der Einfluss; (2) Die von Ökonomen eingenommenen politischen Positionen müssen mit denjenigen von Vertretern anderer Wissenschaften (vor allem Juristen) verglichen werden.

Im Frühjahr 1999 hat der Autor 58 KollegInnen aus 11 Ländern informell gebeten, ihm Informationen über Ökonomen als Politiker zukommen zu lassen. Um die Suche handhabbar zu machen, wurden drei Einschränkungen vorgenommen: (a) Der Begriff "Ökonom" wird auf vollamtliche Professoren (v.a. Ordinarien) der Volkswirtschaftslehre begrenzt; (b) Nur Positionen als 


\section{Was bewirkt die Volkswirtschaftslehre?}

Staatspräsident, als Minister in der Zentralregierung und als Zentralbankpräsidenten werden berücksichtigt. Ökonomen als Parlamentsabgeordnete und als Regierungsmitglieder aus Gliedstaaten werden ausgeklammert. ${ }^{15}$ Ebenso wurde blosse Beratungstätigkeit ausgeschlossen; (c) Es wird nur die Nachkriegszeit betrachtet.

Einige der erhaltenen Antworten waren sehr ausführlich. ${ }^{16}$ In vielen Fällen (vor allem bei Amerikanern) wurde bei den Antworten nicht zwischen Beratungstätigkeit und Exekutiv-Funktionen unterschieden. Bemerkenswert viele Kollegen gestanden, keine Beispiele nennen zu können.

Tabelle 1 gibt eine auf dieser Grundlage gewonnene Auswahl von Ökonomieprofessoren als Politiker (darunter nur eine Frau). Die Ergebnisse beanspruchen keine Vollständigkeit; sicherlich gibt es in vielen Ländern noch weitere Fälle, die aufzuführen wären. ${ }^{17}$ Einigermassen umfassend erscheinen mir die Ergebnisse für Deutschland, die Schweiz, die Niederlande, Belgien, Portugal und Dänemark. Für andere Länder werden nur besonders bemerkenswerte Fälle aufgeführt.

Gemäss dieser Tabelle hat die ökonomische Zunft im engeren Sinne (Professoren) in der Nachkriegszeit eine erkleckliche Zahl von hohen politischen Positionen errungen. Einige unter ihnen haben zweifellos die Wirtschaft und Gesellschaft wesentlich beeinflusst, allen voran ist Ludwig Erhard zu nennen, dem als Wirtschaftsminister die entscheidende Tat zur Einführung der Sozialen Marktwirtschaft in Deutschland zu verdanken ist. Bei den meisten anderen aufgeführten Ökonomen ist jedoch offen, ob sie einen feststellbaren Einfluss ausgeübt haben, und wenn ja, ob er dem damaligen ökonomischen Wissen entsprach und ob der Einfluss - aus heutiger Sicht - für die Wirtschaft und Gesellschaft günstig oder ungünstig einzuschätzen ist.

\section{Einschätzungen aus persönlicher Sicht}

Wie stark beeinflusst die Volkswirtschaftslehre die Wirtschaft und Gesellschaft? Die bisherigen Erörterungen geben kein eindeutiges Bild. Manche dürften den Schluss ziehen, unsere Wissenschaft bewirke viel, während einige andere wesentlich skeptischer sind oder sogar jeglichen Einfluss abstreiten. Aus diesem Grund sei mir zum Abschluss eine Einschätzung aus persönlicher

15. Damit werden einige prominente Ökonomen-Politiker wie Gerhard Zeitel und Paul Senf (beide Finanzminister im Saarland) oder Ernst Buschor und Hans Künzi (beide Regierungsräte, d.h. Minister, im Kanton Zürich) ausgenommen.

16. An dieser Stelle bedanke ich mich insbesondere bei Charles Beat Blankart, Gottfried Bombach, Eric van Damme, H.P. van Dalen, John Flemming, Francesco Forte, René Frey, Gérard Gäfgen, Victor Ginsburgh, Charles Goodhart, Edward Gramlich, Beat Gygi, Bruno Heyndels, Reimut Jochimsen, Jerry Jordan, Erhard Kantzenbach, Mervyn King, Gebhard Kirchgässner, Roger Middleton, Gianfranco Mossetto, Ewald Nowotny, Martin Paldam, Sir Alan Peacock, Ana Bela Santos, Vitor Santos, Friedrich Schneider, Robert Solow, Hannelore Weck-Hannemann.

17. Der Autor ist für weitere Hinweise dankbar (bsfrey@iew.unizh.ch). 


\section{Bruno S. Frey}

Tabelle 1 Ökonomieprofessoren als Politiker (Stand Mitte 1999)

\begin{tabular}{|c|c|c|}
\hline Länder & Personen & Ressort / Position (Amtszeit) \\
\hline \multicolumn{3}{|l|}{ A. Deutschland } \\
\hline \multirow[t]{5}{*}{$\begin{array}{l}\text { Bundes- } \\
\text { minister }\end{array}$} & Erhard, Ludwig ${ }^{1}$ & $\begin{array}{l}\text { Wirtschaft (1949-63); Bundeskanzler } \\
\quad(1963-66)\end{array}$ \\
\hline & Schiller, Karl & Wirtschaft und Finanzen (1966-72) \\
\hline & Töpfer, Klaus & Umwelt (1987-94); Bauten (1994-99) \\
\hline & Müller-Armack, Alfred ${ }^{2}$ & Wirtschaft (1952-57) \\
\hline & Hankel, Wilhelm ${ }^{3}$ & Wirtschaft (1968-72) \\
\hline \multirow{8}{*}{$\begin{array}{l}\text { Zentralbank- } \\
\text { präsidenten }\end{array}$} & Schlesinger, Helmut ${ }^{3}$ & Bundesbank (1991-93) \\
\hline & Tietmeyer, Hans ${ }^{3}$ & Bundesbank (1991-99) \\
\hline & Hesse, Helmut & $\begin{array}{l}\text { Landeszentralbank (LZB) Niedersachsen; } \\
\text { Bremen (1988-98) }\end{array}$ \\
\hline & Jochimsen, Reimut & LZB Nordrhein-Westfalen (1990- ) \\
\hline & Kloten, Norbert & LZB Baden-Württemberg (1976-92) \\
\hline & Krupp, Hans-Jürgen & $\begin{array}{l}\text { LZB Hamburg, Mecklenburg-Vorpommern } \\
\text { und Schleswig-Holstein (1993- ) }\end{array}$ \\
\hline & Sievert, Olaf & LZB Sachsen und Thüringen (1993-98) \\
\hline & Pfleiderer, Otto ${ }^{3}$ & LZB Baden-Württemberg (1948-76) \\
\hline \multirow[t]{2}{*}{ B. Österreich ${ }^{4}$} & Kamitz, Reinhard & $\begin{array}{l}\text { Finanzen (1952-60); Präsident der } \\
\text { Nationalbank (1960-68) }\end{array}$ \\
\hline & Koren, Stephan & $\begin{array}{l}\text { Finanzen (1968-78); Präsident der } \\
\text { Nationalbank (1978-88) }\end{array}$ \\
\hline \multicolumn{3}{|l|}{ C. Schweiz ${ }^{5}$} \\
\hline \multirow[t]{2}{*}{$\overline{\text { Bundesräte }}$} & Weber, Max & Finanzen und Zoll (1951-53) \\
\hline & Deiss, Joseph & Äusseres (1999- ) \\
\hline \multirow[t]{2}{*}{ Nationalbank } & Gehrig, Bruno & Generaldirektor (1996- ) \\
\hline & Schaller, François & Präsident des Bankrates (1986-89) \\
\hline \multirow[t]{8}{*}{ D. Niederlande } & Zijlstra, Jelle & $\begin{array}{l}\text { Wirtschaft (1952-59); Finanzen (1959-63); } \\
\text { Ministerpräsident (1966-67); Präsident } \\
\text { der Zentralbank (1967-81) }\end{array}$ \\
\hline & Lubbers, Ruud & Ministerpräsident (1982-94) \\
\hline & De Quay, E.J. & $\begin{array}{l}\text { Verteidigung (1945/46); Ministerpräsident } \\
\quad \text { (59-63) }\end{array}$ \\
\hline & Andriessen, J.E. & $\begin{array}{l}\text { Finanzen (1989-94); Strasse/Wasserwege } \\
\quad(94-98)\end{array}$ \\
\hline & Duisenberg, Wim & $\begin{array}{l}\text { Finanzen (1973-77); Präsident der } \\
\text { Zentralbank (1982-97) }\end{array}$ \\
\hline & Lieftinck, Piet & Finanzen $(1945-58)$ \\
\hline & Pronk, Jan P. & $\begin{array}{l}\text { Entwicklung und Zusammenarbeit } \\
\quad(1973-77,89-98) ; \text { Umwelt (1998- ) }\end{array}$ \\
\hline & Ritzen, Jo & $\begin{array}{l}\text { Erziehung (1989-94); Erziehung, Kultur } \\
\text { und Wissenschaft (1994- ) }\end{array}$ \\
\hline
\end{tabular}




\begin{tabular}{|c|c|c|}
\hline Länder & Personen & Ressort / Position (Amtszeit) \\
\hline & Witteveen, H.J. & Finanzen (1967-71) \\
\hline & Zalm, Gerrit & Finanzen (1994- ) \\
\hline \multirow[t]{5}{*}{ E. Belgien } & Eyskens, Mark & $\begin{array}{l}\text { Finanzen (1980/81; 81-88); } \\
\text { Ministerpräsident (1981) }\end{array}$ \\
\hline & Coppé, Albert & $\begin{array}{l}\text { Wirtschaft (1950-51); öffentl. Bauten } \\
\quad(1950-52)\end{array}$ \\
\hline & Hulpiau, Raphaël & Gesundheit (1966-68) \\
\hline & Van Offelen, Jacques & $\begin{array}{l}\text { Wirtschaft (1958-60); Industrie/Handel } \\
\text { (1960-61) }\end{array}$ \\
\hline & Simonet, Henri & Wirtschaft (1972); Äusseres (1977-80) \\
\hline \multirow[t]{12}{*}{$\underline{\text { F. Portugal }}$} & Cavaco Silva, Aníbal & $\begin{array}{l}\text { Finanzen und Planung (1980-81); } \\
\text { Ministerpräsident (1985-95) }\end{array}$ \\
\hline & Salazar, Antonio ${ }^{6}$ & Ministerpräsident (1932-68) \\
\hline & Barbosa Pinto, António & Finanzen (1955-65); Gouverneur der ZB \\
\hline & Beleza, Miguel & Finanzen (1990-91); Gouverneur der ZB \\
\hline & Braga de Macedo, Jorge & Finanzen $(1991-93)$ \\
\hline & Mateus, Augusto & Wirtschaft (1996-97) \\
\hline & Moura Pína, Joaquim & Wirtschaft (1997- ) \\
\hline & Lopes, Ernâni & Finanzen und Planung (1983-85) \\
\hline & Lopes da Silva, José & Finanzen und Planung $(1974-75 ; 1978)$ \\
\hline & Nunes, Jacinto & $\begin{array}{l}\text { Finanzen (1978-79); Wirtschaft; } \\
\text { Gouverneur der ZB }\end{array}$ \\
\hline & $\begin{array}{l}\text { Pereira de Moura, } \\
\text { Francisco }\end{array}$ & Wirtschaft (1975) \\
\hline & Salgueiro, João & Finanzen und Planung (1981-83) \\
\hline \multirow[t]{4}{*}{ G. Dänemark } & Andersen, Poul & $\begin{array}{l}\text { Europa (1968-71); Wirtschaft (1968-71; } \\
\quad 73-75 \text { ) }\end{array}$ \\
\hline & Kristensen, Thorkil & Finanzen $(1945-47)$ \\
\hline & Olson, Erling & Wohnungsbau \\
\hline & Philip, Kjeld & Handel (1957-60); Finanzen (1960-65) \\
\hline \multicolumn{3}{|c|}{ H. Andere Länder: Einige herausragende Personen ${ }^{7}$} \\
\hline \multirow[t]{7}{*}{ Italien } & Einaudi, Luigi & $\begin{array}{l}\text { Finanzen (1945-48); Staatspräsident } \\
\quad(1948-55)\end{array}$ \\
\hline & Fanfani, Amintore & $\begin{array}{l}\text { Inneres (1953-54); Ministerpräsident } \\
\quad(1958-59)\end{array}$ \\
\hline & Prodi, Romano & Ministerpräsident (1996-99) \\
\hline & Forte, Francesco & $\begin{array}{l}\text { Finanzen (1982-83); Europa (83-85); } \\
\text { Auslandshilfe (85-87) }\end{array}$ \\
\hline & Martino, Antonio & Äusseres (1994-95) \\
\hline & Reviglio, Franco & Finanzen (1992-93) \\
\hline & Spaventa, Luigi & Finanzen (1993-94) \\
\hline
\end{tabular}




\section{Bruno S. Frey}

Tabelle 1 fortgesetzt

\begin{tabular}{|c|c|c|}
\hline Länder & Personen & Ressort / Position (Amtszeit) \\
\hline \multirow{4}{*}{$\begin{array}{l}\text { Vereinigtes } \\
\text { Königreich }^{8}\end{array}$} & Wilson, Harold & Premierminister (1964-70; 1974-76) \\
\hline & Dalton, Hugh & $\begin{array}{l}\text { Krieg (1940-42); Handel (1942-45); } \\
\quad \text { Finanzen (1945-47); Planung (1950-51) }\end{array}$ \\
\hline & Gaitskell, Hugh & $\begin{array}{l}\text { Brennstoffversorg. (1947-50); Finanzen } \\
\quad(1950-51) ; \text { Leader der Opposition } \\
(1955-63)\end{array}$ \\
\hline & Jay, Douglas & $\begin{array}{l}\text { Handel (1964-67); Präsident des Board of } \\
\text { Trade }\end{array}$ \\
\hline \multirow{4}{*}{$\begin{array}{l}\text { Vereinigte } \\
\text { Staaten }\end{array}$} & Burns, Arthur & Präsident des Federal Reserve (1970-77) \\
\hline & Blinder, Alan S. & $\begin{array}{l}\text { Vizepräsident des Federal Reserve } \\
\text { (1994-96) }\end{array}$ \\
\hline & Wallich, Henry & Board of Governors, Fed. Res. (1974-88) \\
\hline & Schultz, George & $\begin{array}{l}\text { Arbeit (1968-70); Finanzen (1972-74); } \\
\text { Äusseres (1982-89) }\end{array}$ \\
\hline \multirow[t]{2}{*}{ Israel } & Bruno, Michael & Governeur der Bank of Israel (1986-91) \\
\hline & Frenkel, Jacob & Governeur der Bank of Israel (1991- ) \\
\hline \multirow[t]{2}{*}{ Frankreich } & Barre, Raymond & Ministerpräsident (1976-81) \\
\hline & $\begin{array}{l}\text { Strauss-Kahn, } \\
\text { Dominique }\end{array}$ & Industrie (1991-93); Wirtschaft (1997- ) \\
\hline Griechenland & Papandreou, Andreas & Ministerpräsident (1981-89; 1993-96) \\
\hline Türkei & Ciller, Tansu & Ministerpräsidentin (1993-96) \\
\hline \multirow[t]{2}{*}{ Polen } & Lange, Oskar & Vizepräsident \\
\hline & Balcerowicz, Leszek & $\begin{array}{l}\text { Finanzen und Stellv. Ministerpräsident } \\
\text { (1989-91) }\end{array}$ \\
\hline \multirow[t]{2}{*}{ Schweden } & Ohlin, Bertil & Handel (1944-45) \\
\hline & Myrdal, Gunnar & Handel (1945-47) \\
\hline \multirow{2}{*}{$\begin{array}{l}\text { Tschecho- } \\
\text { slowakische } \\
\text { /Tschechische } \\
\text { Republik }\end{array}$} & Sik, Ota & $\begin{array}{l}\text { Wirtschaft und Vizeministerpräsident } \\
\text { (1968) }\end{array}$ \\
\hline & Klaus, Vaclav & $\begin{array}{l}\text { Finanzen (1989-92); Ministerpräsident } \\
\text { (1992-97) }\end{array}$ \\
\hline Argentinien & Cavallo, Domingo & Wirtschaft (1991-96) \\
\hline \multicolumn{3}{|c|}{ I. International } \\
\hline Europäische & Delors, Jacques & Präsident (1985-94) \\
\hline \multirow{2}{*}{ Kommission } & Prodi, Romano & Präsident (1999- ) \\
\hline & Monti, Mario & Mitglied (1995-) \\
\hline Europäische & Duisenberg, Wim & Präsident (1998- ) \\
\hline Zentralbank & Issing, Otmar & Mitglied des Direktoriums (1998- ) \\
\hline
\end{tabular}


Was bewirkt die Volkswirtschaftslehre?

\begin{tabular}{|c|c|c|}
\hline Länder & Personen & Ressort / Position (Amtszeit) \\
\hline $\begin{array}{l}\text { Europäische } \\
\text { Investitionsbank }\end{array}$ & Nowotny, Ewald & Vize-Präsident (1999- ) \\
\hline $\begin{array}{l}\text { UNO- } \\
\text { Finanz- } \\
\text { institutionen }\end{array}$ & $\begin{array}{l}\text { Fischer, Stanley } \\
\text { Stiglitz, Joseph }\end{array}$ & $\begin{array}{l}\text { First Deputy Director, IWF (1994- ) } \\
\text { Senior Vice President, Weltbank (1997- ) }\end{array}$ \\
\hline $\begin{array}{l}1 \text { Seit } 1947 \text { Hono } \\
2 \text { Staatssekretär; } \\
\text { Gerhard Weisser, } \\
{ }^{3} \text { Honorarprofess } \\
\text { verbunden sind. } \\
\text { Leonhard Gleske } \\
{ }^{4} \text { Als Staatssekreta } \\
\text { Nationalrat Alexa } \\
\text { stechen Joseph Sc } \\
5 \text { Ausserdem vers } \\
\text { Schmid, Rémy Scl } \\
6 \text { Professor für Re } \\
7 \text { Entweder hohe } \\
8 \text { Akademische Ö } \\
\text { House of Lords: } \\
\text { John Maynard Ke } \\
\text { besteht gegenwär } \\
\text { (Exekutiv-Direkto }\end{array}$ & $\begin{array}{l}\text { rarprofessor an der U1 } \\
\text { yegen seiner besonder } \\
\text { Heinz Haller, Manfre } \\
\text { ren, die aber beson } \\
\text { Verschiedene Direkt } \\
\text { und Hermann Remsp } \\
\text { ire sind z.B. zu nenner } \\
\text { nder van der Bellen, } \\
\text { humpeter und Eugen } \\
\text { chiedene Mitglieder } \\
\text { heurer und Peter Tsch } \\
\text { cht und öffentliche F } \\
\text { Funktionen und/oder } \\
\text { konomen, aber nich } \\
\text { homas Balogh, Peter } \\
\text { ynes, Lionel Robbins. }\end{array}$ & $\begin{array}{l}\text { Tünchen. } \\
\text { tung aufgeführt. Weitere Staatssekretäre sind z.B. } \\
\text { ermann, Otto Schlecht, Johann Eekhoff. } \\
\text { mit der Volkswirtschaftslehre als Wissenschaft } \\
\text { Bundesbank wie Otmar Issing, Claus Köhler, } \\
\text { ren zuvor Ordinarien der Volkswirtschaftslehre. } \\
\text { eidl und Adolf Nussbaumer, als Abgeordnete zum } \\
\text { kesch und Ewald Nowotny. Aus der Vorkriegszeit } \\
\text { hm-Bawerk hervor. } \\
\text { onalrates, z.B. Franz Jaeger, Remigio Ratti, Hans } \\
\text { (nicht für Volkswirtschaftslehre). } \\
\text { Wissenschaft bekannte Autoren. } \\
\text { soren sind (waren) z.B. folgende Mitglieder des } \\
\text { Meghnad Desai, Richard Kahn, Nicholas Kaldor, } \\
\text { netary Policy Committee der Bank von England } \\
\text { n: Mervyn King (Deputy Governor), John Vickers } \\
\text { Alan Budd, Willem Buiter, Charles Goodhart. }\end{array}$ \\
\hline
\end{tabular}

Sicht erlaubt, so wie ich sie aus der Beschäftigung mit dem gestellten Thema gewonnen habe.

\subsection{Wenig Wissen über den Einfluss der Volkswirtschaftslehre}

Viele Beiträge zum Thema beruhen auf Vermutungen, gewagten Spekulationen oder auf reinem Wunschdenken. Als empirische Evidenz werden häufig ausgewählte Einzelereignisse, und dazu noch oft in anekdotischer Form, angeführt. Überzeugende empirische Belege für einen systematischen und wesentlichen Einfluss der Volkswirtschaftslehre auf Wirtschaft und Gesellschaft fehlen.

Dieser Beitrag möchte dazu anregen, dieses Defizit zu überwinden und die „Ökonomik der Ökonomik" als relevantes Gebiet zu etablieren. Notwendig sind nicht nur empirische Belege, sondern zuvor eine analytische Durchdringung des sicherlich schwierigen Themas.

\subsection{Verabschiedet sich die Volkswirtschaftslehre von der Wirtschaftspolitik?}

Die Volkswirtschaftslehre entwickelt sich immer stärker zu einer Analyse formaler und selbst-definierter Probleme. Diese werden nicht als Antwort auf 
die Herausforderungen der Realität gesehen, sondern richten sich nach den im bestehenden Wissenschaftsdiskurs üblichen intellektuellen Standards. Ein Anspruch auf wirtschaftspolitische Relevanz wird erst gar nicht erhoben. Die gestellte Frage über den gesellschaftlichen Einfluss der Volkswirtschaftslehre wird entsprechend weder gesehen noch als relevant eingeschätzt. Aus dieser Sicht betrachtet dient der Formalismus der Volkswirtschaftslehre als Mittel zur Auswahl unter Bewerbern für eine wissenschaftliche Laufbahn, d.h. sie erfüllt eine Selektionsfunktion. Die Beherrschung des formalen Instrumentariums dient als Signal, dass die entsprechende Person die Fähigkeit für die akademische ökonomische Lehre und Forschung besitzt und damit ein zertifiziertes Mitglied der Zunft werden kann. Dieser nicht einfach zu erwerbende Eintrittspreis stellt ähnliche intellektuelle Anforderungen wie die Fähigkeit von Bewerbern für eine Beamtenkarriere im kaiserlichen China, die schöne Gedichte verfassen können mussten, oder die Beherrschung des Lateins, das bis weit in dieses Jahrhundert als Bildungsnachweis diente.

Diese Sicht der Volkswirtschaftslehre betrifft nicht die gesamte Disziplin. Schwer zu bestreiten ist jedoch, dass dieses Verständnis (a) einen zunehmend grossen Teil der akademischen Volkswirtschaftslehre umfasst und (b) innerhalb der Disziplin als besonders prestigereich gilt. Die Auswirkungen auf die Ausrichtung des wissenschaftlichen Nachwuchses und die Berufungspraxis sind deutlich sichtbar.

\subsection{Wohin steuert die Volkswirtschaftslehre?}

Die sich immer stärker selbst-definierten intellektuellen Problemen zuwendende Volkswirtschaftslehre ist mit einigen ernsthaften Schwierigkeiten konfrontiert, die in der Zukunft sich noch verschärfen dürften:

a) Die Anteile an den Studierenden aller Niveaus nimmt ab, ganz besonders im Vergleich zur Betriebswirtschaftslehre, aber auch zu andern Fächern. Es stellt sich die Frage, ob unser Fach für Spitzenbegabungen noch attraktiv ist. Noch bis vor kurzem haben gerade besonders motivierte junge Leute unser Fach studiert, weil sie zur Lösung gesellschaftlicher Probleme wie Arbeitslosigkeit, Armut oder Umweltproblemen beitragen wollten. Dieser Anreiz geht verloren, wenn sich die Disziplin hauptsächlich oder ausschliesslich mit internen Puzzles herumschlägt.

b) Ressourcen vor allem in Form von Lehrstühlen und Assistentenstellen gehen verloren. Die Volkswirtschaftslehre könnte damit zu einem (wohl unbedeutenden) Teil der angewandten Mathematik werden. Ein derartiger Ressourcenverlust ist für eine Wissenschaft, die im wesentlichen Marktbewertungen akzeptiert, besonders ernst zu nehmen.

c) Die Volkswirtschaftslehre büsst ihre Bedeutung vor allem in den Medien, in der wirtschaftspolitischen Diskussion, aber auch im allgemeinen gesellschaftlichen Diskurs ein. 


\section{Was bewirkt die Volkswirtschaftslehre?}

\subsection{Wo werden die Herausforderungen der Gesellschaft ökonomisch analysiert?}

Wer gerade führende wissenschaftliche Zeitschriften unseres Faches durchgeht, findet immer wieder hervorragende problemorientierte Beiträge. Sie versuchen damit zumindest die Wirtschaft und Gesellschaft zu beeinflussen. Gleichzeitig ist jedoch festzustellen:

a) Wirtschaftliche Probleme werden zunehmend in akademischen Bereichen ausserhalb der volkswirtschaftlichen Lehr- und Forschungseinrichtungen erstellt. Immer häufiger finden sich besonders wichtige Beiträge in der Betriebswirtschaftslehre (Business Administration), Rechtswissenschaft, Geografie, Soziologie, Politikwissenschaft oder Public Policy. Entsprechend wird bereits die entsprechende Ausbildung in diesen Bereichen unternommen.

b) Ein zunehmender und heute bereits beträchtlicher Teil der problemorientierten Forschung wird in gewinnorientierten Firmen und in gemeinnützigen wissenschaftlichen Netzwerken unternommen. Sie sind ausserhalb der Universitäten angesiedelt und die Verknüpfung mit der akademischen Lehre geht weitgehend verloren.

c) Schliesslich entwickelt sich immer stärker eine „Alltagsökonomie“, derer sich vor allem Journalisten, Politiker und andere Meinungsmacher bedienen und die bei einer Niederschrift nicht selten in von Millionen gelesenen Bestsellern resultiert. Diese Art von Volkswirtschaftslehre ist meilenweit vom heute dominierenden Diskurs in unserem Fach entfernt: Gerade formalen Modellen verpflichtete Vertreter unseres Faches sind hierbei sprachlos. Damit wird die Ausbreitung dieser von der akademischen Volkswirtschaftslehre abgekoppelten Vorstellungen gefördert.

Die verschiedenen aufgezeigten Konsequenzen sind für Vertreter einer nicht an den Herausforderungen der Gesellschaft orientierten Volkswirtschaftslehre belanglos. Für andere jedoch - und dazu zählt sich auch der Autor - sind diese Aussichten unerfreulich.

\section{$\overline{\text { Literaturverzeichnis }}$}

Aaron, Henry J. (1987), Symposium on Tax Reform. Economic Perspectives 1, 7-10.

Acs, Zoltan J., David B. Audretsch und Maryann P. Feldman (1991), Real Effects of Academic Research: Comment. American Economic Review 82, 363-367.

Allen, G.C. (1979), The British Disease: a short essay on the nature and causes of the nation's lagging wealth. IEA, London.

Alston, R.M., J.R. Kearl und M.B. Vaughan (1992), Is there a consensus among economists in the 1990s? American Economic Review 82 (2, Papers \& Proceedings), 203-209. 


\section{Bruno S. Frey}

Baron, James, N. und Michael T. Hannan (1994), The Impact of Economics on Contemporary Sociology, in: Richard Swedberg (ed.). Economic Sociology. Elgar, Cheltenham, 530-65.

Barro, R.J. (1993), Council of economic irrelevance. Wall Street Journal.

Becker, Gary S. (1985), Public policies, pressure groups, and dead weight costs. Journal of Public Economics 28, 371-400.

Blankart, Charles B. (1981), Towards an Economic Theory of Advice and Its Application to the Deregulation Issue. Kyklos 34, 95-105.

Blaug, Mark (1997), Ugly Currents in Modern Economics. Fact or Fiction? Conference on Realism in Economics, Rotterdam, 14-15 November.

Blendon, Robert J. et al. (1997), Bridging the Gap Between the Public's and Economists' View of the Economy. Journal of Economic Perspectives 11, 105-118.

Blinder, Alan S. (1997), Distinguished Lecture on Economics in Government: What Central Bankers Could Learn from Academics - and Vice Versa. Journal of Economic Perspectives 11, 3-19.

Bommer, Rolf und Heinrich W. Ursprung (1998), Spieglein, Spieglein an der Wand. Eine publikationsanalytische Erfassung der Forschungsleistungen volkswirtschaftlicher Fachbereiche in Deutschland, Österreich und der Schweiz. Zeitschrift für Wirtschaftsund Sozialwissenschaften 118 (1), 1-28.

Bonus, Holger (1982), Information und Emotion in der Politikberatung - Zur politischen Umsetzung eines wirtschaftstheoretischen Konzepts. Zeitschrift für die gesamte Staatswissenschaft 138, 1-21.

Borner, Silvio (1975), Wissenschaftliche Ökonomik und politische Aktion: Eine Politische Ökonomie der professionellen Beratung der Wirtschaftspolitik. Haupt, Bern.

Brittan, S. (1973), Is there an Economic Consensus?: An Attitude Survey. Macmillan, London.

Brittan, S. (1995), Economics and Ethics. In: S. Brittan und A. Hamlin (Hrsg.). Market Capitalism and Moral Values. Edward Elgar, Aldershot, 1-22.

Carter, J. und M. Irons (1991), Are Economists Different, and If So, Why? Journal of Economic Perspectives 5, 171-177.

Cassidy, J. (1996), The Decline of Economics. New Yorker, 2. Dezember, 50-60.

Clower, Robert W. (1989), The State of Economics: Hopeless but not Serious? In: David Colander und A.W. Coats (Hrsg). The Spread of Economic Ideas. Cambridge University Press, Cambridge, 23-41.

Coase, Ronald H. (1999), Economists and Public Policy. In: Daniel B. Klein (ed.). What Do Economists Contribute? New York University Press, New York, 33-52.

Coats, Alfred W. (1981), Economists in Government: An International Comparative Study. Duke University Press, Durham, N.C.

Coats, Alfred W. (1986), Economists in International Agencies: An Exploratory Study. Praeger, New York.

Coats, Alfred W. (1989), Economic Ideas and Economists in Government: Accomplishments and Frustrations. In: David C. Colander und A. W. Coats (Hrsg). The Spread of Economic Ideas. Cambridge University Press, Cambridge, 109-118.

Coats, Alfred W. und David C. Colander (1989), An Introduction to the Spread of Economic Ideas. In: David C. Colander und A. W. Coats (Hrsg.). The Spread of Economic Ideas. Cambridge University Press, Cambridge, 1-19.

Colander, David und Arjo Klamer (1987), The Making of an Economist. Journal of Economic Perspectives 1, 95-111.

Colander, David C. (1989), The Invisible Hand of Truth. In: David C. Colander und A. 


\section{Was bewirkt die Volkswirtschaftslehre?}

W. Coats (Hrsg). The Spread of Economic Ideas. Cambridge University Press, Cambridge, 31-36.

Cordes, Joseph J., Arjo Klamer und Thomas C. Leonard (1993), Academic Rhetoric in the Policy Arena: The Case of Capital Gains Taxation. Eastern Economic Journal 19, 459479.

Dalen, Harry P. van und Arjo Klamer (1997), Blood is Thicker than Water: Economists and the Tinbergen Legacy. In: Peter A.G. van Bergeijk, A. Lans Bovenberg, Eric E.C. van Damme und Jarig van Sinderen (Hrsg). Economic Science and Practice: The Roles of Academic Economists and Policy-makers. Edward Elgar, Cheltenham, 60-91.

Demsetz, H. (1997), The Primacy of Economics: An Explanation of the Comparative Success of Economics in the Social Sciences. Economic Inquiry 35, 1-11.

Economist (1997), The Puzzling Failure of Economics. 23 August 1997, 13.

Ehrenberg, Ronald G. (1999), The Changing Distribution of New Ph.D. Economists and Their Employment: Implications for the Future. Journal of Economic Perspectives 13, 135-138.

Eichenberger, Reiner, Rolf Arpagaus und Ursina Meier (1999), Ökonomen, Publikationen und Zitationen: Ein europäischer Vergleich. Jahrestagung der Schweizerischen Gesellschaft für Statistik und Volkswirtschaft, 25. und 26. März, Fribourg.

Faulhaber, Gerald R. und William Baumol (1988), Economists as Innovators. Journal of Economic Literature 26, 577-600.

Feldstein, Martin (1997), The Council of Economic Advisers: From Stabilization to Resource Allocation. American Economic Review 87, 99-106.

Frank, Robert H. und Philip J. Cook (1995), The Winner-Take-All Society. Free Press, New York.

Frank, Robert H., Thomas D. Gilovich und Dennis R. Regan (1993), Does Studying Economics Inhibit Cooperation? Journal of Economic Perspectives 7, 159-171.

Frey, Bruno S. und Reiner Eichenberger (1992), Economics and Economists: A European Perspective. American Economic Review 82 (May), 216-220.

Frey, Bruno S. und Reiner Eichenberger (1993), American and European Economics and Economists. Journal of Economic Perspectives 9 (1) (Winter), 203-212.

Frey, Bruno S. und Reiner Eichenberger (1997), Economists: First Semester, High Flyers, and UFOs. In: Peter A. G. van Bergejik, A. Lans Bovenberg, Eric E. C. Van Damme und Jarig van Sinderen (Hrsg.). Economic Science and Practice: The Roles of Academic Economists and Policy-makers. Edward Elgar, Cheltenham, UK and Brookfield, USA, $15-48$.

Frey, Bruno S. und Gebhard Kirchgässner (1994), Demokratische Wirtschaftspolitik. Vahlen, München.

Frey, Bruno S., Werner W. Pommerehne und Beat W. Gygi (1993), Economics Indoctrination or Selection? Some Empirical Results. Journal of Economic Education 24, 271-281.

Frey, Bruno S., W.W. Pommerehne, F. Schneider und G. Gilbert (1984), Consensus and dissension among economists: an empirical inquiry. American Economic Review 74 (5), 986-994.

Frey, Bruno S. und Alois Stutzer (1999), Measuring Preferences by Subjective Well-Being. Journal of Institutional and Theoretical Economics 155, erscheint demnächst.

Frey, Bruno S., Werner, W. Pommerehne, Friedrich Schneider und Hannelore Weck (1982), Welche Ansichten vertreten Schweizer Ökonomen? Ergebnisse einer Umfrage, Schweizerische Zeitschrift für Volkswirtschaft und Statistik, 118, 1-40. 


\section{Bruno S. Frey}

Frey, René L. (1996), Ökonomie und Politik: Über die Schwierigkeit der wirtschaftspolitischen Beratung. Rektoratsrede; Basler Universitätsreden 29. Heft. Basel, Helbing \& Lichtenhahn.

Frey, René L. und Bruno S. Frey (1995), Is there a European Economics? Kyklos 48 (Special Issue), 187-311.

Friedman, Milton (1972), Have Monetary Policies Failed? American Economic Review 62, Papers \& Proceedings, 11-18.

Friedman, Milton (1986), Economists and Economic Policy. Economic Inquiry 14 (January), 1-10.

Fuchs, Victor R., Alan B. Krueger und James M. Poterba (1997), Why do Economists Disagree about Policy? The Roles of Beliefs about Parameters and Values, NBER Working Paper No. 6151.

Gäfgen, G. (1976), Politische Ökonomie und Lehre von der Wirtschaftspolitik: Zur Realisierung wirtschaftspolitischer Vorschläge. In: H. Körner, P. Meyer-Dohm, E. Tuchtfeld und C. Uhlig (Hrsg.). Wirtschaftspolitik - Wissenschaft und politische Aufgabe. Bern / Stuttgart, 123-143.

Galbraith, James (1988), The Grammar of Political Economy. In: Arjo Klamer, D. McCloskey und R. Solow (Hrsg). The Consequences of Economic Rhetorik. Cambridge University Press, New York, 221-39.

Galbraith, J.K. (1975), Letter. The Times, 12.

Hahn, F.H. (1985), In praise of economic theory. University College London, London.

Hamilton, L.H. (1992), Economists as Public Policy Advisers. Journal of Economic Perspectives 6 (Summer), 61-64.

Hansen, J. Lee (1999), The Link from Graduate Education in Economics to the Labor Market. Journal of Economic Perspectives 13, 147-152.

Hayek, Friedrich A. (1991), On Being an Economist. In: W.W. Bartley und Stephen Kresge (Hrsg.). The Trend of Economic History. University of Chicago Press, Chicago, $35-48$.

Hirschman, Albert O. (1982), Shifting Involvements. Private Interests and Public Action. Martin Robertson, Oxford.

Jaffe, Adam B. (1989), Real Effects of Academic Research. American Economic Review 79, 957-970.

Jöhr, Walter A. und Hans W. Singer (1955), The Role of the Economist as Official Advisor. Allen and Unwin, London.

Jones, Philip R. und John G. Cullis (1993), Public choice and public policy: The vulnerability of economic advice to the interpretation of politicians. Public Choice 75 , 63-77.

Joskow, Paul L., Richard Schmalensee und Elisabeth M. Bailey (1998), The Market for Sulfur Dioxide Emissions. American Economic Review 88, 669-685.

Kearl, J.R., C.L. Pope, B.C. Whiting und L.T. Wimmer (1979), A Confusion of Economists? American Economic Review, Papers \& Proceedings 69, $28-37$.

Keynes, J. M. (1936), The General Theory of Employment Interest and Money. Macmillan, London.

Kirchgässner, Gebhard (1993), Vom Nutzen der Wirtschaftstheorie für die Wirtschaftspolitik. Konjunkturpolitik 39, 201-225.

Kirchgässner, Gebhard (1996), Ideologie und Information in der Politikberatung: Einige Bemerkungen und ein Fallbeispiel. Hamburger Jahrbuch für Wirtschafts- und Gesellschaftspolitik 41, 11-41.

Kirchgässner, Gebhard (1998), Wirtschaftspolitische Beratung aus der Sicht des 


\section{Was bewirkt die Volkswirtschaftslehre?}

kritischen Rationalismus: vierzehn Thesen. Karl Poppers kritischer Rationalismus, Gummersbach.

Kirchgässner, Gebhard (1999), On the political economy of policy advice. In: Ernst Mohr (ed.). The Transfer of Economic Knowledge. Edward Elgar, Cheltenham, 13-31.

Klamer, Arjo und David Colander (1990), The Making of an Economist. Westview Press, Boulder, Col.

Klamer, Arjo und Jennifer Meehan (1999), The crowding out of academic economics: The case of Nafta. Mimeo, Dept. of Economics, University of Rotterdam.

Klein, Daniel B. (ed.) (1999), What do Economists Contribute? New York University Press, New York.

König, Heinz (1999), Economic Knowledge Transfer by Research Institutes in Germany: Some Reflections. In: Ernst Mohr (ed.). The transfer of economic knowledge. Edward Elgar, Cheltenham, 90-99.

Krueger, Anne O. (1999), Implications of the Labor Market for Graduate Education in Economics. Journal of Economic Perspectives $\overline{13,153-156 .}$

Krueger, Anne O. et al. (1991), Report of the Commission on Graduate Education in Economics. Journal of Economic Literature 29, 1035-1053.

Krugman, Paul R. (1995), Incidents from my Career. In: Arnold Heertje (ed.). The Makers of Modern Economics. Vol. 2. Edward Elgar, Aldershot UK.

Krugman, Paul (1996), Pop internationalism. Cambridge, MA, MIT Press.

Laband, David N. und Richard O. Beil (1999), Are economists more selfish than other „social“ scientists? Public Choice 100, 85-101.

Leontief, W. (1971), Theoretical Assumptions and Nonobserved Facts. American Economic Journal 61, 1-7.

Magee, Stephen P. (1992), The Optimum Number of Lawyers: A Reply to Epp. Law and Social Inquiry 17, 667-693.

Mandel, Michael J. (1999), Going for the Gold: Economists as Expert Witnesses. Journal of Economic Perspectives 13, 113-120.

Marshall, Alfred (1890), The Principles of Economics. 8th. edn. (1920), London, Mcmillan.

Mayer, Thomas (1993), Truth versus Precision in Economics. Edward Elgar, Aldershot.

McCloskey, Donald (1983), The Rhetoric of Economics. Journal of Economic Literature (June), 481-547.

Meier, Alfred und Daniel Mettler (1988), Theorie der Wirtschaftspolitik und wirtschaftspolitische Beratung. In: Praxisorientierte Volkswirtschaftslehre. Stämpfli, Bern.

Meier, Ursina B. (1994), Wissenschaftliche Publikationsaktivitäten an Hochschulen - ein Vergleich der volkswirtschaftlichen Abteilungen deutschweizer Hochschulen. Schweiz. Zeitschrift für Volkswirtschaft und Statistik 130, 207-232.

Middleton, Roger (1998), Charlatans or Saviours? Economists and the British Economy from Marshall to Meade. Edward Elgar Publisher, Northhampton MA.

Nelson, R.H. (1987), The Economics Profession and the Making of Public Policy. Journal of Economic Literature 25, 49-91.

Peacock, A. T. (1988), An Economic Analysis of Economic Advice-Giving. Atlantic Economic Journal 16, 1-10.

Peacock, A. T. (1991), Economic advice and economic policy. In: D. Greenaway, M. Bleaney und I.M.T. Stewart (Hrsg.). Companion to Contemporary Economic Thought. Routledge, London, 713-716.

Peacock, Alan T. (1992), The Credibility of Economic Advice to Government. Economic Journal 102, 1213-1222. 


\section{Bruno S. Frey}

Peacock, A.T. (1994), The Utility Maximizing Government Economic Adviser: Comment. Public Choice 80, 191-197.

Peters, Guy und Anthony Barker (1993), Advising West European Gevernments: Inquiries, Expertise and Public Policy. Edinburgh University Press, Edinburgh.

Pommerehne, Werner W., Friedrich Schneider, Guy Gilbert und Bruno S. Frey (1984), Concordia Discors: Or: What do Economists Think? Theory and Decision 16, 251-308.

Posner, Richard A. (1999), The Law and Economics of the Economic Expert Witness. Journal of Economic Perspectives 13, $\overline{91}-100$.

Prendergast, Canice (1999), The Provision of Incentives in Firms. Journal of Economic Perspectives 37, 7-63.

Rivlin, A. (1987), Economics and the Political Process. American Economic Review 77, 110.

Samuels, Warren J. (1980), Economics as a Science and Its Relation to Policy: The Example of Free Trade. Journal of Economic Issues 14 (March), 163-85.

Schelling, Thomas (1997), Why does economics only help with easy problems? In: Peter A.G. van Bergeijk, A. Lans Bovenberg, Eric E.C. van Damme und Jarig van Sinderen (Hrsg). Economic Science and Practice: The Role of Academic Economists and Policy-makers. Edward Elgar, Cheltenham, 134-148.

Schlecht, Otto und Ulrich van Suntum (Hrsg) (1995), 30 Jahre Sachverständigenrat zur Begutachtung der gesamtwirtschaftlichen Entwicklung. Sinus, Krefeld.

Schneider, Friedrich, Werner W. Pommerehne und Bruno S. Frey (1983), Relata referimus: Eine Befragung deutscher Ökonomen. Zeitschrift für die gesamte Staatswissenschaft 139 (März), 19-66.

Schneider, Hans-Karl (ed.) (1968), Grundsatzprobleme wirtschaftspolitischer Beratung. Berlin, Schriften des Vereins für Socialpolitik.

Schultze, Charles L. (1996), The CEA: An Inside Voice for Mainstream Economics. Journal of Economic Perspectives 10, 23-39.

Shleifer, Andrei (1998), State versus Private Ownership. Journal of Economic Perspectives $12,133-150$.

Siegenthaler, Hansjörg (1993), Regelvertrauen, Prosperität und Krisen: die Ungleichmässigkeit wirtschaftlicher und sozialer Entwicklung als Ergebnis individuellen Handelns und sozialen Lernens. Mohr, Tübingen.

Siegfried, John J. (1998), Trends in Undergraduate Economics Degrees: A 1996-97 Update. Journal of Economic Education 29, 285-288.

Siegfried, John J. und Wendy A. Stock (1999), The Labor Market für New Ph.D. Economists. Journal of Economic Perspectives 13 (3), 115-134.

Stein, Herbert (1996), A Successful Accident: Recollections and Speculations about the CEA. Journal of Economic Perspectives 10 (3), 3-21.

Stigler, G. J. (1982), The Economist as Preacher and Other Essays. The University of Chicago Press, Chicago.

Stigler, George J. (1984), Economics - The Imperial Science? Scandinavian Journal of Economics 86, 301-313.

Stiglitz, Joseph (1997), Looking Out for the National Interest: The Principles of the Council of Economic Advisers. American Economic Review 87, 109-113.

Stiglitz, Joseph (1998), Distinguished Lecture on Economics in Government: The Private Uses of Public Interests: Incentives and Institutions. Journal of Economic Perspectives 12, 3-22.

Thornton, Robert und John Ward (1999), The Economist in Tort Litigation. Journal of Economic Perspectives 13, 101-112. 


\section{Was bewirkt die Volkswirtschaftslehre?}

Towse, Ruth und Mark Blaug (1988), The Current State of the British Economics Profession. RES, London.

Tullock, Gordon (1984), How to Do Well While Doing Good! In: David C. Colander (ed.). Neoclassical Political Economy: The Analysis of Rent-Seeking and DUP Activities. Cambridge, Mass., Ballinger, 229-240.

Von Beckerath, Erwin und Herbert Giersch (Hrsg) (1963), Probleme der normativen Ökonomik und der wirtschaftspolitischen Beratung. Schriften des Vereins für Socialpolitik, Berlin.

Weber, Luc (1982), Les „Trois Sages“: une necessité ou un anachronisme? Schweizerische Zeitschrift für Volkswirtschaft und Statistik 118, 41-57.

Williamson, J. (1994), In search of a manual for technopols. In: J. Williamson (ed.). The political economy of policy reform. Institute for International Economics, Washington, DC, 11-28.

Williamson, J. (1997), Comments. In: A.W. Coats (ed.). The Post-1945 Internationalization of Economics. Duke University Press, Durham, NC.

Wirtschaftswoche (1997), Der Einfluss der Ökonomen auf die deutsche Wirtschafts- und Finanzpolitik wird immer geringer - mit fatalen Folgen. Nr. 38, 36-43.

Wittman, Donald (1989), Why Democracies Produce Efficient Results. Journal of Political Economy 97 (December), 1395-1424.

Wyplosz, C. (1999), The Culture of Economic Policy Advice: An International Comparison with Special Emphasis on Europe. In: E. Mohr (Hg.) The Transfer of Economic Knowledge, Edward Elgar, Cheltenham, 47-73.

Zimmermann, Heinz (1999), Financial innovation, the transfer of knowledge, and implications for postgraduate education. In: Ernst Mohr (ed.). The Transfer of Economic Knowledge. Edward Elgar, Cheltenham, 125-150.

Abstract: Due to its formality and highly analytic thinking, economics is often attributed a leading role among the social sciences and a prominent position as contributor to economic or social issues in the real world. Fact is, however, that the empirical proof for such a claim is either missing or anecdotal.

This paper aims to outline the 'economics of economics'. It surveys and compares approaches of impact measurement such as a production function of economics or the demand and supply of trained economists. It furthermore discriminates between the impact of economic ideas versus that of economists as scientists or politicians. 